\title{
Amperometric sensing of hydrogen peroxide vapor for security screening
}

\author{
John Benedet • Donglai Lu • Karel Cizek • \\ Jeff La Belle • Joseph Wang
}

Received: 31 December 2008 /Revised: 30 March 2009/Accepted: 1 April 2009/Published online: 24 April 2009

(C) The Author(s) 2009. This article is published with open access at Springerlink.com

\begin{abstract}
Rapid detection of the hydrogen peroxide precursor of peroxide explosives is required in numerous security screening applications. We describe a highly sensitive and selective amperometric detection of hydrogen peroxide vapor at an agarose-coated Prussian-blue (PB) modified thick-film carbon transducer. The sensor responds rapidly and reversibly to dynamic changes in the level of the peroxide vapor, with no apparent carry over and with a detection limit of $6 \mathrm{ppbv}$. The remarkable selectivity of the PB-based screen-printed electrode towards hydrogen peroxide leads to effective discrimination against common beverage samples. For example, blind tests have demonstrated the ability to selectively and non-invasively identify concealed hydrogen peroxide in drinking cups and bottles. The attractive performance of the new microfabricated PBbased amperometric peroxide vapor sensor indicates great potential for addressing a wide range of security screening and surveillance applications.
\end{abstract}

Keywords Hydrogen peroxide · Prussian-blue . Vapor sensor · Screen-printed electrodes · Explosives . Security screening

K. Cizek $\cdot$ J. Wang $(\bowtie)$

Department of Nanoengineering,

University of California-San Diego,

La Jolla, CA 92093-0448, USA

e-mail: josephwang@ucsd.edu

J. Benedet $\cdot$ D. Lu $\cdot$ J. La Belle

The Biodesign Institute and Departments of Chemical

Engineering and Chemistry and Biochemistry,

Arizona State University,

Tempe, AZ 85287-5801, USA

\section{Introduction}

Growing security concerns have generated urgent needs for innovative tools for field screening homemade explosives and their precursors [1]. Particular recent attention has been given to peroxide explosives (particularly triacetone triperoxide (TATP)) due to their widespread use by terrorists and their straightforward synthesis from readily available hydrogen peroxide and acetone [2]. In numerous security screening applications, it is crucial to detect rapidly the hydrogen peroxide precursor both in the liquid and vapor phases. Eliasson et al. [3] reported recently on the use of Raman Spectroscopy for detecting hydrogen peroxide concealed in bottles/containers. In another recent study, Bohrer et al. [4] described the use of phthalocyanine-based chemiresistors for the vapor-phase detection of hydrogen peroxide.

Here, we report on the use of a Prussian-blue (PB)-based thick-film amperometric sensor for rapid and selective detection of hydrogen peroxide vapor. Electrochemical devices have shown considerable promise for on-site detection of explosives [5]. Such devices have been used previously for environmental monitoring of atmospheric hydrogen peroxide $[6,7]$ but not towards security screening of peroxide vapor. We demonstrated recently an "AddDetect" electrochemical approach for detecting solid and liquid samples of the peroxide explosives TATP and hexamethylene triperoxide diamine based on the electrocatalytic detection of their acid-generated hydrogen-peroxide product at a PB-modified electrode [8]. Prussian-blue acting as "artificial peroxidase" offers a highly selective, preferential low-potential electrocatalytic detection of hydrogen peroxide [9]. It has been widely used for liquid-phase peroxide measurements but rarely for direct vapor analysis and not towards security screening applications. 
In the following sections, we illustrate that an agarosecoated PB-modified screen-printed electrode (SPE) sensor offers a rapid, highly sensitive, and selective response to hydrogen peroxide vapor. Screen-printed (thick-film) electrochemical sensors are readily produced at low cost and are extremely attractive for decentralized testing [10]. The potential of the new PB-screen-printed sensor for security screening applications is demonstrated by the sensor's ability to distinguish non-invasively and rapidly concealed hydrogen peroxide from common beverages in drinking cups and other containers. Such high selectivity of the PBbased vapor electrochemical sensor holds great promise for minimizing false alarms common to airport screening or other threat-detection applications.

\section{Experimental section}

Instrumentation Chronoamperometric measurements were performed using a CHI 1030 Electrochemical Analyzer (CH Instruments, Austin, TX, USA) along with screenprinted electrode transducers. Gas dilutions were performed using a 1010 Precision Gas Diluter with an internal pump, Tedlar ${ }^{\mathrm{TM}}$ bags, Bev-A-Line tubing $(6.5 \mathrm{~mm}$ o.d $\times 3.12 \mathrm{~mm}$ i. d.) and JACO polypropylene connectors (Custom Sensor Solutions, Oro Valley, AZ, USA). A semi-automatic screen printer (Model TF 100; MPM, Franklin, MA, USA) was used for printing the thick-film Prussian-blue modified carbon (working), carbon (counter), and $\mathrm{Ag} / \mathrm{AgCl}$ (pseudo reference) electrodes. The carbon and silver inks [Ercon, E3449 graphite and $\mathrm{R}-2412 \mathrm{Ag} / \mathrm{AgCl}$, respectively] were printed through a patterned stencil on $10 \times 10 \mathrm{~cm}$ ceramic plates containing 30 strips $(3.3 \times 1.0 \mathrm{~cm}$ each $)$. All three electrodes were cured at $150^{\circ} \mathrm{C}$ for $1 \mathrm{~h}$. The insulating ink (Ercon, E6165-116, Blue Insulayer) was subsequently printed on a portion of the plate, leaving $2 \times 2 \mathrm{~mm}$ sections on both ends, defining the electrode area on one side and the silver electrical contacts (of the three electrodes) on the opposite end. The insulating layer was cured at $100^{\circ} \mathrm{C}$ for $1 \mathrm{~h}$.

Reagents Stock solutions of hydrogen peroxide were prepared by diluting a $30 \%(w / w) \mathrm{H}_{2} \mathrm{O}_{2}$ standard solution purchased from Mallinckrodt (Phillipsburg, USA). Deionized water from a Milli-Q system (Millipore, Bedford, MA, USA) was used to prepare all solutions. Potassium ferricyanide, iron (III) chloride, graphite powder, potassium chloride, and agarose I-A were all purchased from Sigma-Aldrich (St Louis, USA). Hydrochloric acid (12 M) was obtained from EMD (Darmstadt, Germany).

Preparation of the PB-modified ink The PB-modified ink was prepared using the following procedure. One gram of graphite powder and $10 \mathrm{~mL} 0.1 \mathrm{M}$ iron (III) chloride were mixed with stirring for $2 \mathrm{~min}$. Next, $10 \mathrm{~mL}$ of $0.1 \mathrm{M}$ potassium ferricyanide were added, and the resulting solution was stirred for $10 \mathrm{~min}$. The resulting mixture was filtered, and the filtrand was dried at $100^{\circ} \mathrm{C}$ for $10 \mathrm{~h}$. The resulting $\mathrm{PB}$-coated graphite powder was then mixed with the commercial carbon ink at three different loadings $(2.5 \%, 5.0 \%$, and $10 \% \mathrm{w} / w)$.

Hydrogel solid electrolyte preparation One hundred milligrams of agarose I-A was dissolved under stirring in $5 \mathrm{~mL}$ $0.2 \mathrm{M} \mathrm{KCl}$ solution. The mixture was slowly heated until boiling and kept at that temperature for $5 \mathrm{~min}$. Subsequently, the gel solution was cooled down to around $65^{\circ} \mathrm{C}$ and kept at this constant temperature with stirring (liquid phase). A 50$\mu \mathrm{L}$ agarose droplet was cast on the end of the electrode, covering the three electrode areas. The droplet was allowed to cool to room temperature and rapidly solidified to form a $1-\mathrm{mm}$ thin gel layer covering the three electrodes (over $6 \times$ $10 \mathrm{~mm})$.

Chronoamperometric measurements of $\mathrm{H}_{2} \mathrm{O}_{2}$ at $\mathrm{PB}$ modified SPE To generate hydrogen peroxide vapor, $25 \mathrm{ml}$ glass bottles (Fig. 1, c) containing $100 \mu \mathrm{L}$ solutions of various concentrations of $\mathrm{H}_{2} \mathrm{O}_{2}$ were used (Fig. 1, d). The samples were allowed to equilibrate for $30 \mathrm{~min}$ in the sealed environment. Subsequently, a PB-modified SPE was introduced into the bottle through an opening in its cap (Fig. 1, a) and sealed in place with parafilm. Prior to insertion in the glass vial, a $50-\mu \mathrm{L}$ agarose droplet was cast on the end of the electrode (Fig. 1, b). Current measurements were performed following a 1-min exposure of the electrode to the vapor. A similar procedure was used for testing the commercial beverage samples. Chronoamper-

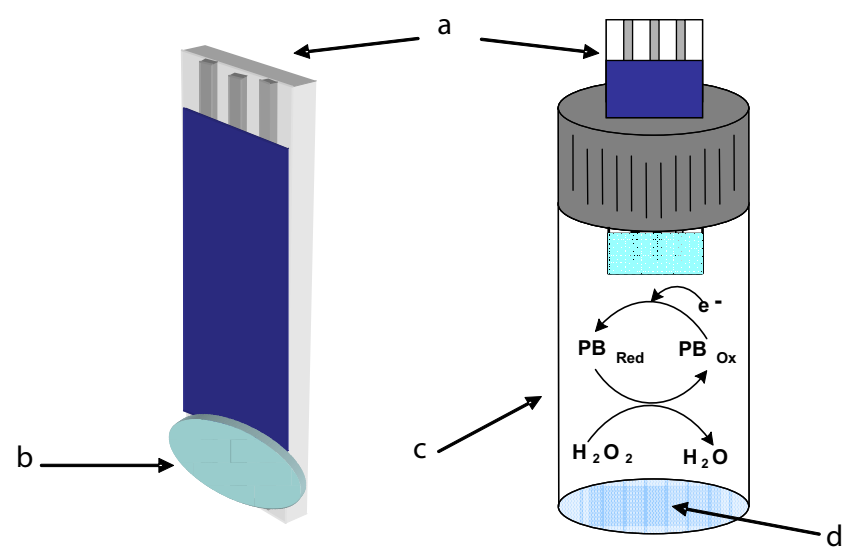

Fig. 1 Sensor and experimental setup for the vapor detection of $\mathrm{H}_{2} \mathrm{O}_{2}$. PB-modified SPE on alumina oxide ceramic $(a)$ coated with $50 \mu \mathrm{L}$ of $2 \%$ agarose I-A in $1 \mathrm{M} \mathrm{KCl}(b)$ as a solid electrolyte. The SPE is placed into $25 \mathrm{ml}$ bottle (c) containing $100 \mu \mathrm{L}$ of various hydrogen peroxide solutions $(d)$ that have been equilibrated with the sealed environment 
ometry with a double potential step was performed by holding the initial potential at $0.2 \mathrm{~V}$ for $20 \mathrm{~s}$ and then stepping it to the measurement potential $(-0.05 \mathrm{~V})$ for 100 s. Quantitative measurements were carried out by sampling the current after $50 \mathrm{~s}$.

Transient measurements were performed in a cylindrical glass tube (internal diameter, $10 \mathrm{~cm}$; total length, $60 \mathrm{~cm}$; Fig. 5). Samples of hydrogen peroxide were placed inside the tube in a plastic weigh boat, and the SPE was inserted through a small slit in the glass tube approximately $50 \mathrm{~cm}$ above the sample. For transient measurements, the initial potential $(0.2 \mathrm{~V})$ was applied for $5 \mathrm{~s}$, then the secondary potential $(-0.05 \mathrm{~V})$ was applied for $35 \mathrm{~s}$, with the current sampling carried out after $30 \mathrm{~s}$. There was a 20 -s interval between runs.

On-line measurements of $\mathrm{H}_{2} \mathrm{O}_{2}$ in a flow system To generate hydrogen peroxide vapor, $1 \mathrm{ml}$ of a $0.0025 \%(w / w) \mathrm{H}_{2} \mathrm{O}_{2}$ was added to a $4 \mathrm{~L}$ Tedlar $^{\mathrm{TM}}$ bag, and the bag was subsequently filled with nitrogen. The sample was allowed to equilibrate for $6 \mathrm{~h}$ in the sealed environment and was then connected via a Bev-A-Line tubing to the gas diluter. A 40-L TedlarTM bag, filled with the nitrogen background gas, was also connected to the gas diluter via Bev-A-Line tubing. The gas diluter was then connected to the flow cell with Bev-A-Line tubing. The electrochemical flow cell comprised a cylindrical glass tube $(2.0 \mathrm{~cm}$ in length, $2.5 \mathrm{~cm}$ i.d.) affixed at the bottom to a ceramic plate that had been modified to allow insertion of the planar thick-film strip electrode. The other end of the flow cell was capped with a rubber stopper connected to the gas diluter and equipped for outlet flow. The stopper was placed approximately $0.75 \mathrm{~cm}$ above the hydrogel-covered PB-modified electrode, resulting in a total cell volume of approximately $3.7 \mathrm{~mL}$. In addition to the $50 \mu \mathrm{L}$ of hydrogel placed directly on the sensing area, an additional hydrogel $(50 \mu \mathrm{L})$ aliquot was placed over the insulator area of the SPE to increase the humidity around the working sensing area. The sample vapor was diluted with nitrogen to alternating concentrations of 25 and $100 \mathrm{ppbv}$, and the flow rate of diluted $\mathrm{H}_{2} \mathrm{O}_{2}$ vapor to the flow cell was maintained at $4.1 \mathrm{scc} / \mathrm{s}$. A PBmodified SPE was inserted into the flow cell for the amperometric measurement of the $\mathrm{H}_{2} \mathrm{O}_{2}$ vapor, carried out at an applied potential of $-0.2 \mathrm{~V}$.

Amperometric measurements of peroxide vapor at $P B$ modified SPE for screening applications To generate hydrogen peroxide vapor, a $10-\mathrm{mL}$ sample of a $30 \% \mathrm{w} /$ $w \mathrm{H}_{2} \mathrm{O}_{2}$ solution was added to a 500-mL Styrofoam coffee cup (covered with a lid) and was allowed to equilibrate for $30 \mathrm{~min}$. The PB-modified SPE was placed at different distances $(0.5-2.5 \mathrm{~cm})$ above the drinking opening in the lid, and quantitative current measurements were taken at an applied potential of $-0.05 \mathrm{~V}$. This potential was applied for $5 \mathrm{~min}$ to ensure a stable current baseline signal before placing the sensor above the lid opening.

\section{Results and discussion}

Factors affecting the chronoamperometric detection of the hydrogen peroxide vapor at the PB-modified SPE have been examined and optimized (Fig. 2). The effect of the loading of the PB-coated graphite powder in the carbon ink upon the hydrogen peroxide reduction current is shown in Fig. 2a. As expected, a higher PB loading in the carbon ink resulted in an increased cathodic current signal. The current increases steadily from $0 \%$ to $5 \%$ PB loading and more rapidly between $5 \%$ and $10 \%$. Above $10 \%$, the consistency of the mixture of carbon ink and PB-coated graphite powder was such that it became impossible to successfully print PB-modified SPEs. Figure $2 b$ displays the effect of the applied potential on the hydrogen peroxide reduction current. The signal increases slowly upon changing the potential from $0.2 \mathrm{~V}$ to $-0.05 \mathrm{~V}$, more rapidly to $-0.1 \mathrm{~V}$, and levels off at more negative potentials. PB-modified SPEs with a $10 \%$ PB loading, operated at an applied potential of $-0.05 \mathrm{~V}$, were thus used for all subsequent chronoamperometric measurements. A thin gel electrode coating, prepared from a $50-\mu \mathrm{L} 2 \%$ agarose I-A droplet (see "Experimental section"), offered and provided the most favorable tradeoff between sensitivity and speed.

Before applying to blind testing, the PB-modified SPE was evaluated in chronoamperometric measurements of different hydrogen peroxide vapors. Figure 3 displays current-time chronoamperometric recordings for hydrogen peroxide vapor generated during a 30-min equilibration

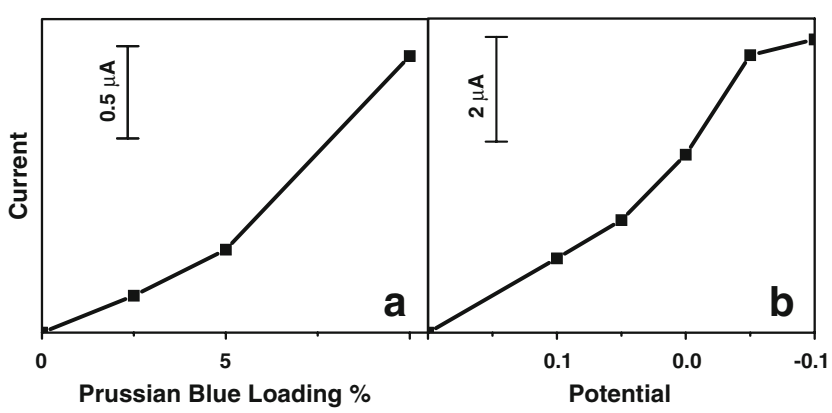

Fig. 2 Optimization of the $\mathrm{H}_{2} \mathrm{O}_{2}$ vapor detection at the PB-modified SPE. a Effect of the PB ink loading and $\mathbf{b}$ of the measurement potential. Vapor generated from $100 \mu \mathrm{L}$ samples of a $3.0 \%(w / w)$ $\mathrm{H}_{2} \mathrm{O}_{2}$ solution in a $25 \mathrm{~mL}$ flask. Current sampling $50 \mathrm{~s}$ after the potential step. Potential (a), $-0.05 \mathrm{~V}$ (vs. pseudo $\mathrm{Ag} / \mathrm{AgCl}$ ); $\mathrm{PB}$ loading (b), 10\% $(w / w)$. Other conditions, as in Fig. 1 


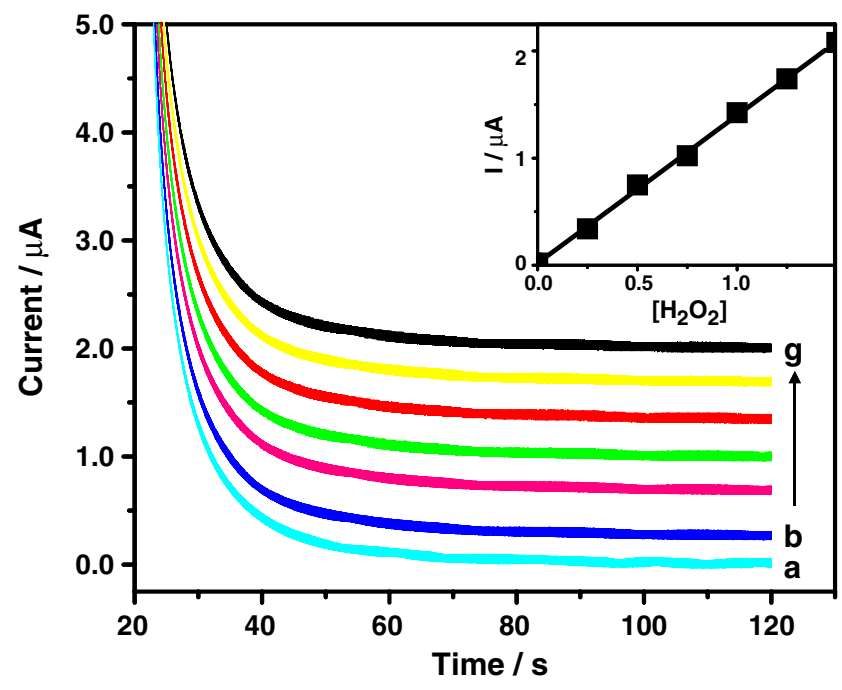

Fig. 3 Chronoamperometric response of water (blank) vapor (a) and of vapors from aqueous solutions containing increasing levels of $\mathrm{H}_{2} \mathrm{O}_{2}$ in $0.25 \%(w / w)$ steps (curves $b-g)$. Inset shows the corresponding calibration plot of current vs. percent $\mathrm{H}_{2} \mathrm{O}_{2}(w / w)$. Potential step to $-0.05 \mathrm{~V}$ (vs. pseudo $\mathrm{Ag} / \mathrm{AgCl}$ ) with current sampling after $50 \mathrm{~s}$. PB loading, $10 \%(w / w)$. Other conditions, as in Figs. 1 and 2

with $1 \mathrm{~mL}$ droplets containing increasing levels of hydrogen peroxide in $0.25 \%$ increments $(\mathrm{b}-\mathrm{g})$, along with the corresponding background response for water (a). Welldefined chronoamperometric signals are observed for these peroxide concentrations. The current (sampled after $50 \mathrm{~s}$ ) is proportional to the peroxide concentration. The resulting calibration plot (shown in the inset) is highly linear $(R=$ 0.999) with a slope of $1.4 \mu \mathrm{A} /\left[\begin{array}{ll}\% & \mathrm{H}_{2} \mathrm{O}_{2}\end{array}\right]$. While such chronoamperometric measurements offer a detection limit of ca. $0.1 \%(\mathrm{LOD}=3 \mathrm{~N})$; a dramatically lower detection limit of ca. $0.008 \%$ hydrogen peroxide was observed in the continuous amperometric measurements (discussed below).

The specificity of the PB-modified SPE sensor towards hydrogen peroxide vapor is crucial for potential security screening applications. Successful detection of the hydrogen peroxide precursor (at concentrations greater than $10 \%$ $w / w$, used for preparing peroxide explosive devices) is desired in an airport screening setting. The ability to selectively and non-invasively detect the presence of hydrogen peroxide vapor in common beverage containers is critical for such screening. Such selectivity has been demonstrated by performing blind tests on a wide variety of common beverages. Such ability to selectively and noninvasively identify hydrogen peroxide vapor when hidden among numerous common beverages is illustrated in Fig. 4. When tested against eight other common beverages, a welldefined chronoamperometric signal was observed only for the hydrogen peroxide sample (E). Apparently, none of the numerous constituents of the different drinks is responding at the PB-based SPE (A-D, F-I). No false positives or negatives were observed in multiple repetitions of these data $(n=10)$. These results confirm that the remarkable selectivity of the PB amperometric sensor [9] leads to an effective discrimination against common beverage samples. Such high specificity offers great promise for minimizing false alarms in airport screening operations.

The ability of the PB-modified SPE to detect hydrogen peroxide vapor at a relatively large distance from its source was investigated using the experimental setup shown in Fig. 5 (right). This test involved exposure to a peroxide vapor generated from a $1-\mathrm{mL}$ droplet of hydrogen peroxide $(30 \% \mathrm{w} / \mathrm{w})$ placed at a distance of $50 \mathrm{~cm}$ (i.e., at the bottom of the glass tube). Such test led to a well-defined
Fig. 4 Chronoamperometric response of different beverage samples: bottled drinking water $(A)$, apple juice $(B)$, Coke $(C)$, iced coffee $(D), 10 \% \mathrm{H}_{2} \mathrm{O}_{2}(E)$, milk $(F)$, beer $(G)$, gin $(H)$, and iced tea $(I)$. Other conditions, as in Figs. 1 and 3

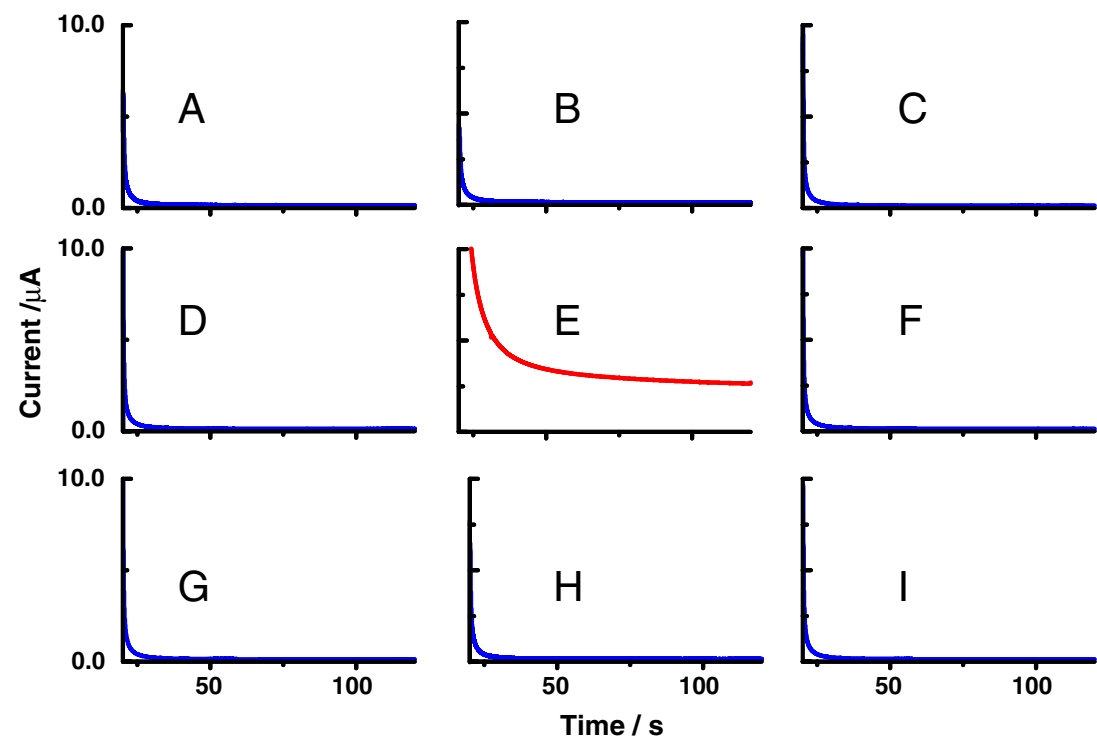


chronoamperometric signal for hydrogen peroxide within 2 min (Fig. 5, c). Longer exposure times led to even higher sensitivity (Fig. 5, d-f).

The response and recovery times of the PB-modified SPE detector were evaluated using alternate 1-min exposures to hydrogen peroxide vapor pulses of 25 and 100 ppbv (Fig. 6). The measured current increases rapidly, approaching its steady-state signal within $40 \mathrm{~s}$ after the onset of the exposure, before the end of the exposure. The recovery time of the sensor is even faster, with the current returning to its baseline level within nearly $30 \mathrm{~s}$. Such repetitive exposures led to a highly reproducible response, reflecting the absence of memory effects. The fast and sensitive response are coupled with high sensitivity, with an estimated detection limit of 6 ppbv $(\mathrm{LOD}=3 \mathrm{~N})$. Such detection limit compares favorably to those reported for other electrochemical hydrogen peroxide vapor sensors based on voltammetric [11] and amperometric [12-15] transductions.

In an attempt to mimic potential real-life security applications of the sensor, a second set of blind tests was performed for identifying hydrogen peroxide concealed in a standard coffee cup with a lid (using the experimental setup of Fig. 7, inset). The SPE sensor was thus placed ca. $2.5 \mathrm{~cm}$ above the opening of the lid to allow monitoring of the "escaping" peroxide vapor. For the cup containing hydrogen peroxide (Fig. 7, D), the amperometric signal began to increase rapidly approximately $25 \mathrm{~s}$ after placing the sensor over the lid opening. No false positives were observed for any of the other common beverages (tested in Fig. 7, A-C). Similarly, no false negatives were observed for subsequent measurements of hydrogen peroxide $(n=10)$. The absence

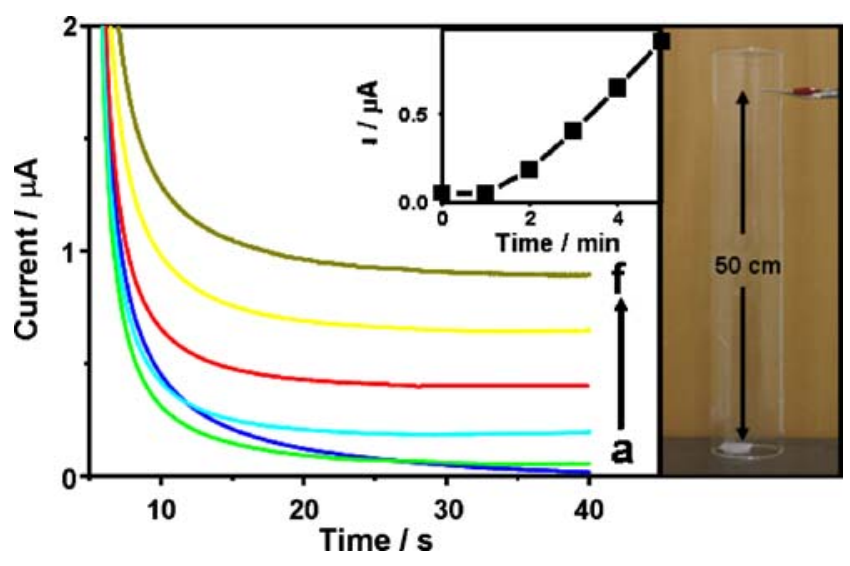

Fig. 5 Chronoamperometric response at the PB-modified SPE for a $1 \mathrm{~mL}$ droplet of $30 \% \mathrm{H}_{2} \mathrm{O}_{2}$ solution in a glass tube. The electrode was placed $50 \mathrm{~cm}$ above the sample, and measurements were performed at 1-min intervals, from $0(a)$ to $5(f)$ min. Potential step to $-0.05 \mathrm{~V}$ (vs. pseudo $\mathrm{Ag} / \mathrm{AgCl}$ ). Current measured at $30 \mathrm{~s}$ following the potential step. The experimental setup (including the glass tube, the $\mathrm{H}_{2} \mathrm{O}_{2}$ sample and the SPE sensor) is shown on the right

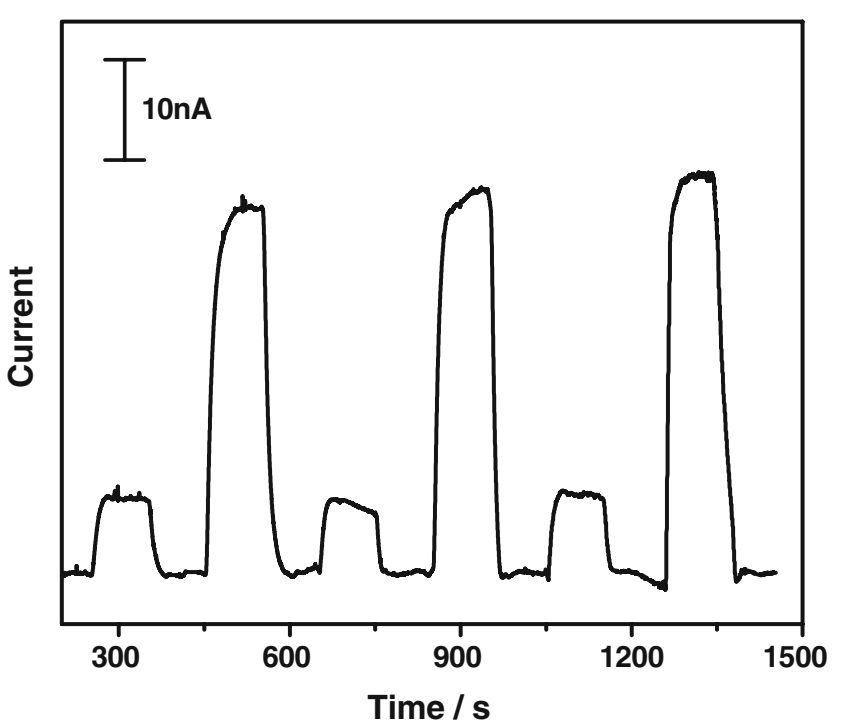

Fig. 6 On-line amperometric measurements of 100 and $25 \mathrm{ppbv}_{2} \mathrm{O}_{2}$ at the PB-modified SPE. Alternate exposure to such 1-min peroxide pulses. Solid electrolyte: $2 \%$ agarose I-A prepared in $0.2 \mathrm{M} \mathrm{KCl}$. Fixed applied potential, $-0.20 \mathrm{~V}$; flow rate, $4.1 \mathrm{cc} / \mathrm{s}$. Carrier (blank) nitrogen

of any false negative results is of vital importance for any security application.

\section{Conclusions}

This article addresses the urgent needs to detect the hydrogen peroxide precursor of peroxide explosives. It demonstrates a

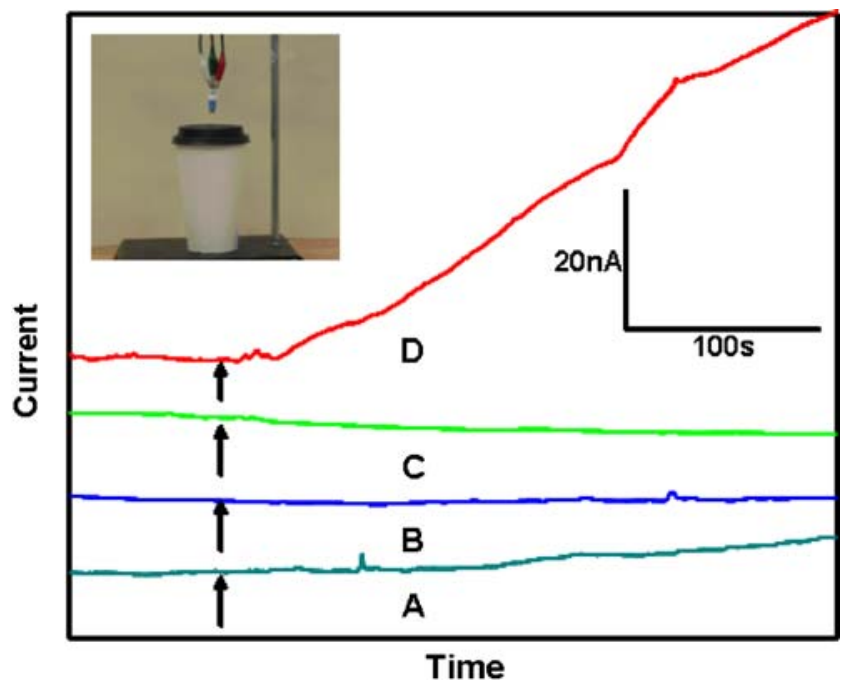

Fig. 7 Non-invasive screening of beverages in drinking cups for concealed $\mathrm{H}_{2} \mathrm{O}_{2}$. Amperometric response of the PB-modified SPE to vapors of $(A)$ juice, $(B)$ coffee, $(C)$ water, and $(D) 30 \% \mathrm{H}_{2} \mathrm{O}_{2}$. Onset of exposure to $\mathrm{H}_{2} \mathrm{O}_{2}$ vapor (moving the sensor above the opening in the cup lid) is shown by the arrows. Fixed applied potential $-0.05 \mathrm{~V}$ (vs. pseudo $\mathrm{Ag} / \mathrm{AgCl}$ ); solid electrolyte: $2 \%$ agarose I-A prepared in $0.2 \mathrm{M}$ $\mathrm{KCl}$ 
highly selective and sensitive vapor detection of hydrogen peroxide at an agarose-coated PB-modified screen-printed carbon-electrode transducer. The new hydrogen-peroxide vapor sensor offers reliable non-invasive screening of concealed hydrogen peroxide in drinking cups and is not responding to constituents of a wide range of common beverages. Coupling such remarkable selectivity of the PB modifier, with high sensitivity and speed, as well as with portability and low cost of the SPE, makes this new electrochemical route extremely attractive for addressing a wide range of threat detection field scenarios.

Acknowledgments We wish to thank Motorola Inc. and the US DIA for the financial support.

Open Access This article is distributed under the terms of the Creative Commons Attribution Noncommercial License which permits any noncommercial use, distribution, and reproduction in any medium, provided the original author(s) and source are credited.

\section{References}

1. Yinon Y (2002) Trends Anal Chem 21:292-301

2. Schulte-Ladbeck R, Vogel M, Karst U (2006) Anal Bioanal Chem 386:559-565

3. Eliasson C, Macleod NA, Matousek P (2007) Anal Chem 79:8185-8589

4. Bohrer F, Colesniuc C, Park J, Schuller I, Kummel AC, Trogler WC (2008) J Am Chem Soc 130:3712-3713

5. Wang J (2007) Electroanalysis 19:415-423

6. Huang H, Dasgupta PK, Gonfa Z et al (1996) Anal Chem 88:2062-2066

7. O'Sullivan DW, Lee MY, Noone BC et al (1996) J Phys Chem 100:3241-3247

8. Munoz R, Lu D, Cagan A et al (2007) Analyst 132:560-565

9. Karyakin AA (2001) Electroanalysis 13:813-819

10. Hart JP, Wring SA (1994) Electroanalysis 6:617-624

11. Holmstrom SD, Cox JA (1998) Electroanalysis 9:597-601

12. Kulys J (1992) Sens Actuators B 9:143-147

13. Toniolo R, Geatti P, Bontempelli G et al (2001) J Electroanal Chem 514:123-128

14. Hornok V, Dekany I (2007) J Colloid Interface Sci 309:176-182

15. Huang HL, Dasgupta PK (1997) Talanta 44:605-615 